\title{
The Differentiated Call Processing Based on the Simple Priority-Scheduling Algorithm in SIP6*
}

\author{
Chinchol Kim, Byounguk Choi, Keecheon Kim, Sunyoung Han \\ Department of Computer Science and Engineering, Konkuk University, \\ 1, Hwayangdong, Kwangin-gu, Seoul, 143-701, Korea \\ \{bredkim, buchoi, kckim, syhan\} akonkuk.ac.kr
}

\begin{abstract}
We proposed and implemented a differentiated call processing mechanism in SIP6 (Session Initiation Protocol based on IPv6) implemented by this paper. In order to satisfy the service quality in VoIPv6, call quality and call setup quality should be provided in the Internet. End-to-end QoS for call quality and call setup quality in the VoIP Server like to gatekeeper/gateway, SIP Proxy Server, and SIP Redirect Server are essential. In order to support the end-to-end QoS, RSVP, and DiffServ proposed by IETF have been used. Nevertheless, call setup quality for call processing in VoIP Server has not been supported in the current VoIP systems. To solve this problem, we proposed a concept of differentiated call processing mechanism in SIP6 Server. For differentiating services, we use the Flow Label field of the IPv6 header and the predefined service levels. In this paper, we presented the design and implementation of SIP6. We explain the simple priority-scheduling algorithm that is applied to support QoS call processing. We also demonstrated the better performance by supporting the differentiated call processing service to satisfy the user's required QoS.
\end{abstract}

\section{Introduction}

H.323 and SIP protocol are used for call control in the VoIP technology [9][10]. H.323 has gained the reputation because of its stability and performance through the long time research. H.323 has extensible and flexible because it permits the backward compatibility, E.164 numbers, URLs, TAs, e-mail address, H.323 IDs, and mobile UIMs. But, it has more problem in terms of complexity than SIP [9]. SIP only handles function to establish and control the sessions and has a simple structure providing a good mobility and interaction with other protocols (HTTP, RTSP, and SMTP). As a result, the current Internet society has made the SIP as a standard in many fields such as 3GPP, VoIP, Messaging Systems and ALL-IP system (next generation mobile system). However, H.323 and SIP is do not support the QoS for call processing.

\footnotetext{
* This work is supported by the grant No. R01-2001-00349 from the Basic Research Program of the Korea Science and Engineering Foundation.
} 
VoIP is needs a QoS support for call setup and voice quality. It also requires a security association among peers for authentication. Those requirements can resolved with the IPv6 techniques of Flow Label, AH, and ESP header.

Currently, in order to satisfy the VoIPv6 service quality, QoS for call and call setup quality must be supported in the Internet. In order to support these QoS, the followings are required.

- End-to-end QoS for call quality over the network

- QoS for call setup quality for call processing in VoIP Server during the session establishment.

In order to satisfy the end-to-end QoS, RSVP and DiffServ proposed by IETF have been used. RSVP supports resource reservation mechanism in a local environment and DiffServ supports the differentiated service in the Internet backbone. Nevertheless, In establishing a session, QoS features to meet the call setup quality in VoIP Server cannot be supported by using RSVP and DiffServ. To solve these problems, we propose a concept of differentiated call processing mechanism that uses a simple priority-scheduling algorithm in SIP6. A service level to satisfy the user's required QoS is categorized in to High Quality, Medium Quality, and Normal Quality. A service level is marked in the Flow Label field of the IPv6 header. In this paper, we explain the design and implementation of SIP6 supporting QoS call processing and also demonstrate the better performance resulted from supporting the differentiated call processing service to satisfy the user's required QoS.

This paper presents the system design in section 2. The implementation result is explained in section 3. And the performance analysis is shown in section 4. Finally, we describe the conclusion and future work in section 5.

\section{System Design}

The SIP6 (Session Initiation Protocol based on IPv6) proposed in this paper is a SIP protocol supporting a differentiated call processing mechanism based on the IPv6 protocol. This system consists of SIP6 Server and User Agent. In order to establish a session, two members exchange information through the SIP6 Server.

Fig. 1 shows the system components and the following explains each component.

- SIP6 Daemon contains Proxy Server, Redirect Server, Location Server, and Registrar Server. It refers to a configuration file. QoS Processor receives all IPv6 packets and applies a differentiated call processing for satisfying the user's required QoS.

- Web Registrar Server is responsible for registering the information of user location; it is associated with a user database through the web.

- User Database is responsible for adding, deleting, and updating the user information. 
- SIP6 $\boldsymbol{U A}$ is a SIP6 User Agent. This is divided into two parts: SIP6 User Agent Client for requesting a session and SIP6 User Agent Server for accepting a session. When SIP6 UA Client requests a session and registers the information of user location, SIP6 UA Client marks up the service level for the user's required QoS through the QoS marker.

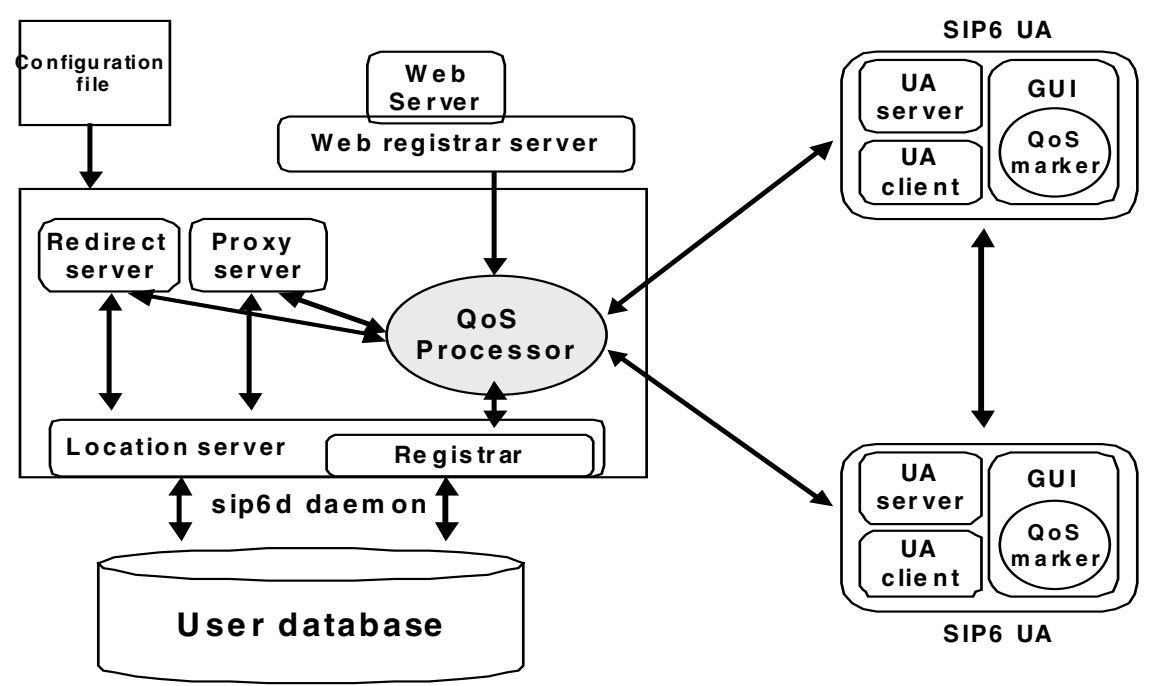

Fig. 1. SIP6 System Components

\subsection{SIP6 Server Design}

SIP6 Server provides the services for user registration, management, user location, call-forward, and call-redirect. It is consisted of QoS Processor and SIP6 Server Module.

\subsubsection{QoS Processor}

QoS Processor applies a differentiated call processing in the session establishment. It defines three service levels (High Quality, Medium Quality, Normal Quality). If QoS Processor receives a packet, Classify Processor classifies the packets according to the service level. Priority-scheduling Processor processes a packet by using a differentiated call processing mechanism. Message Parsing Processor and Method Processor parses the messages and call the SIP6 Server Module. Fig. 2 shows the schedule for processing messages in the QoS Processor.

The Classify Processor is an UDP receiver that receives the SIP6 messages. Those received messages are divided into the Request and Response message types. If the received message is a Request message, the differentiated call processing mechanism is applied. If the received message is a Response message, this message is sent to 
Message Parsing Processor. In the case of Request message, the messages are classified and stored at the High, Medium or Normal Quality Buffer according to the service level specified in the Flow Label field of the IPv6 packet. The service level is defined as follows.

- High Quality has the highest level priority and requires a fast session establishment by using the High Quality Buffer.

- Medium Quality has the middle level priority and uses the Medium Quality Buffer.

- Normal Quality has the lowest level priority and uses the Normal Quality Buffer.

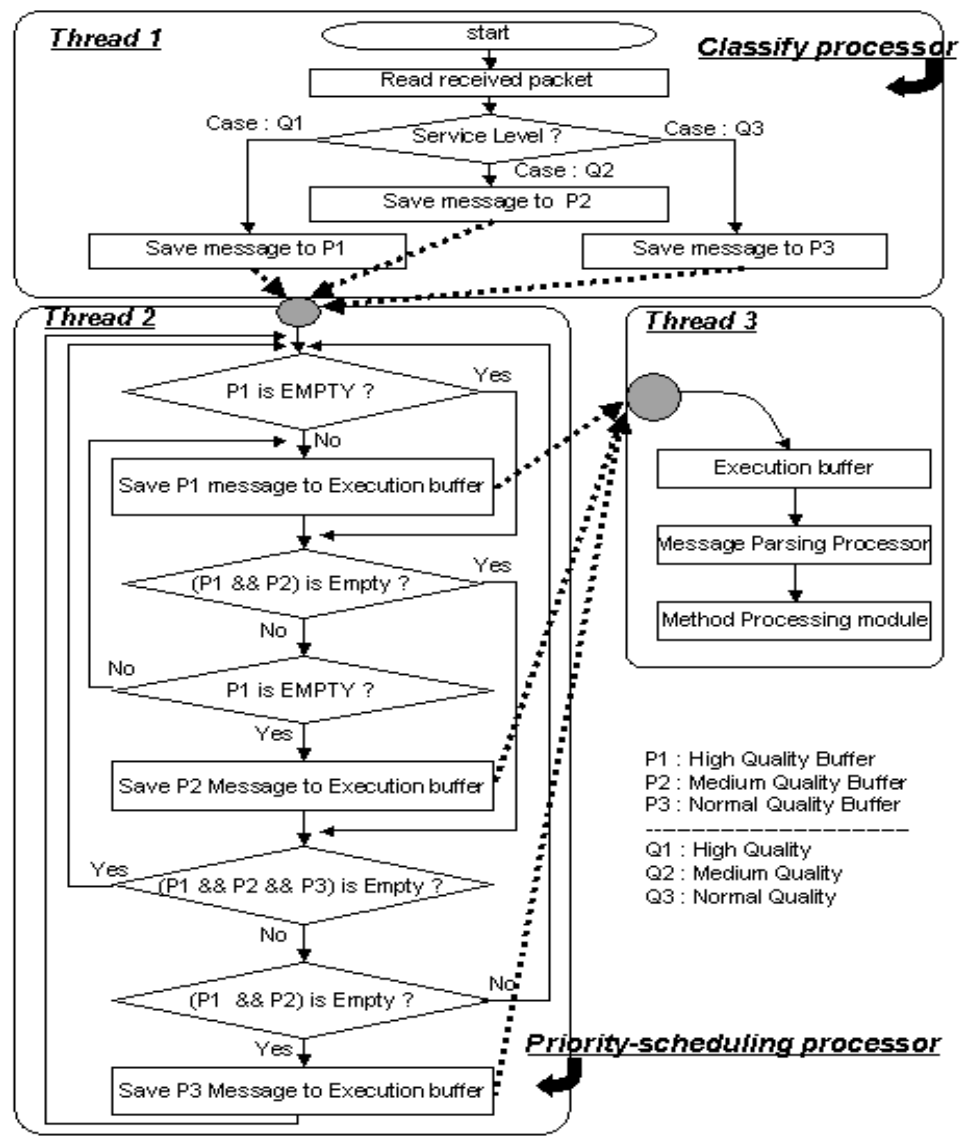

Fig. 2. QoS Processor Procedure

The Priority-scheduling Processor continually examines the messages stored in the multi buffer (High, Medium, Normal Quality Buffer) and applies the differentiated service according to the service levels. It can dynamically process the three buffers at 
a time when a message arrives. Priority-scheduling processor is based on a simple priority-scheduling algorithm. The simple priority-scheduling algorithm operates as follows.

- In the first level priority buffer, this processor directly calls the Message Parsing Processor to process a message.

- After processing the message, it looks for the buffer of next high priority.

- While processing a message in the lower priority buffer, it can be interrupted and handle a message with the higher priority.

By this mechanism, the differentiated call processing service can be supported. Thread 2 in the Fig. 2 shows a flow for the simple priority-scheduling algorithm.

In order to process the message received by Priority-scheduling Processor, the Message Parsing Processor can be divided into several parts.

- Request Message Processing

To avoid the duplicate message, the Request message can be mapped into a unique ID by hash function, and the message with the Request-URI, TO, FROM, Call-ID and Cseq must be stored in the "Execution Buffer". After validating the uniqueness of the message, it can be parsed to detect the errors. At last, Method Processing module is called for the suitable service for the matched methods (BYE, INVITE, OPTIONS, and REGISTER).

- Response Message Processing

It performs a response processing function for a received Request message and a received Response message. In processing a Response message, it sets up a Response Status Code for the Request message. And it generates a socket to send a Response message to the corresponding client

Finally, the Method Processor calls the SIP6 Server Module. In case of INVITE message, it calls Proxy/Redirect Server Module according to the Server Action included in the Request message. In the case of REGISTER message, it calls Registrar Server to register user information. According to RFC 2543, it performs a proper work for OPTION, BYE and CANCEL messages.

\subsubsection{SIP6 Server Module}

SIP6 Server Module is consisted of Proxy Server Module for call forwarding service, Redirect Server Module for call redirect service, and Location Server Module for servicing the user location and registering the user information.

The Proxy Server Module manages the forwarding function for the received calls. Depending on the received methods, it invites a session by forwarding INVITE method after getting user location information from Location Server Module. A transmitted message sets the service timeout during which Proxy Server processes the message. When the timeout occurs, it will cancel the request and send Response 
message with 487 Status Code. If it is an ACK, ACK method is sent to the destination client.

The Redirect Server Module processes the redirect function for the received calls. When it receives INVITE method, it initializes the connection header for the Response message. After setting the service timeout, it gets information of user location from Location Server. If it receives the address as a domain, this module will send URI (User ID, Host, Port number) to the client who has requested INVITE after mapping the IP address through a DNS resolving function of the system.

The Location Server Module is responsible for enrolling the user information and tracking the user location. It includes the Registrar to receive the user enrollment, User Input Table to manage the user ID authorized for accessing the system, User Identity Table to give the multiple IDs per user (So there is a unique user ID used in the session establishment in this table), and User Contact Table to manage the user's current location.

User information from the Registrar can be transformed to the unique user ID that is in the User Identity Table and registered into the User Contact Table. The registered data are used to serve the request of user location from the Proxy and Redirect Server. The user entry information consists of User ID, SIP6 UA port number, URL, Server Action, and Service Level.

\subsection{SIP6 UA Design}

SIP6 UA uses the IPv6 UDP socket interface. This module has GUI for the user interface, UA Client Module and UA Server Module. GUI includes QoS Marker for setting the service levels and interfaces for user registration, session invitation. SIP6 UA Server and Client Module perform the function to send and receive calls in order to establish sessions.

\subsection{Call Processing Procedure and Message}

The SIPv6 suggested in this paper follows the call processing procedure and message formats specified in IETF RFC 2543.

\section{Implementation}

\subsection{SIP6 Implementation}

SIP6 Server is implemented with C language on Linux Kernel 2.2.x supporting the IPv6. The programs are designed to compose the Proxy, Redirect, Location, and Registrar Server. It is executed by daemon process named SIP6d. 
When we start a SIP6 Server, it creates a thread that can open an IPv6 UDP socket and it receives an INVITE Request message. When SIP6d receives a message, the message is classified by checking the service level specified in the Flow Label field of IPv6 header. SIP6d also creates another thread to process the classified Request message sequentially according to the service level proposed by this paper. Finally, According to the method type and Server Action, the thread selectively calls Proxy, Redirect, or Registrar Server Module.

\subsection{QoS Processor Implementation}

We use the Flow Label field of the IPv6 header to specify the service levels in applying the differentiated call processing mechanism. Currently, the Flow Label field is composed of 24-bit to define the QoS. But it has not been used yet. In the Fig. 3, we defined the code values for service levels.

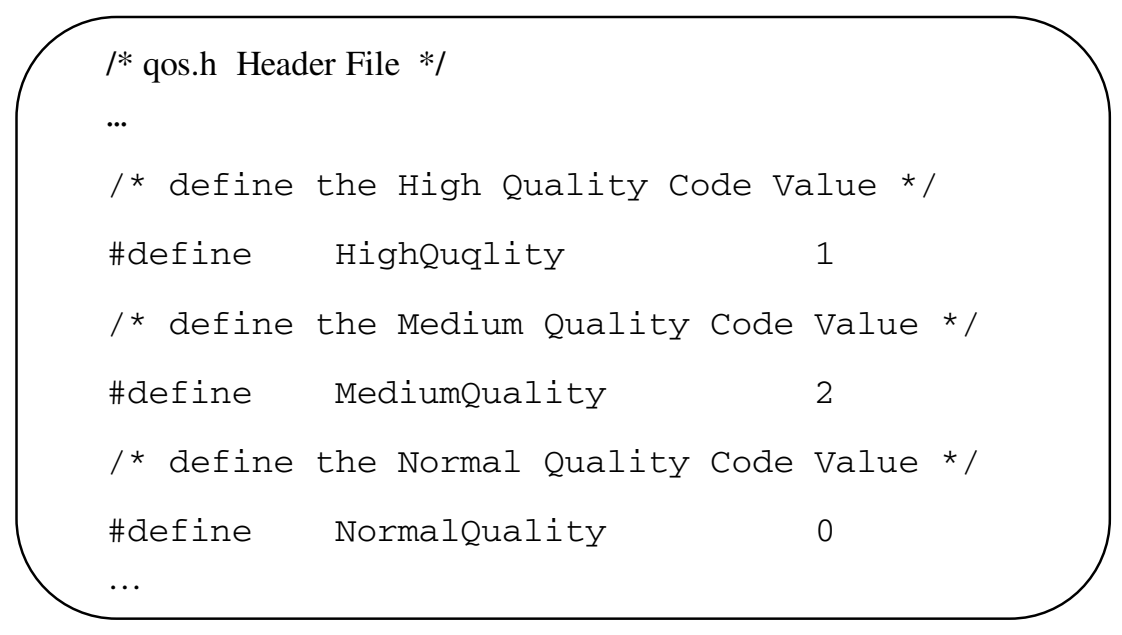

Fig. 3. Code value for service levels defined in qos.h header file

The IPv6 socket structure (struct sockaddr_in6) includes the variable for the Flow Label field in the IPv6 header. In the implementation, the sin6_flowinfo variable in sockaddr_in6 structure is used to specify the code value for the service level.

$\begin{array}{lll}\text { struct } & \text { sockaddr_in6 } & \\ \text { uint8_t } & \text { sin6_len; } \\ & \text { sa_family_t } & \text { sin6_family; } \\ \text { in_port_t } & \text { sin6_port; } \\ \text { uint32_t } & \text { sin6_flowinfo; } & \\ \text { struct } & \text { in6_addr } & \text { sin6_addr; } \\ \text { \} } & & \end{array}$


When a session is established, SIP6 UA specifies a service level in sin6_flowinfo field of socket_in6 structure. If SIP6 Server receives an INVITE Request message, it checks a service level in sin6_flowinfo field and dynamically processes the INVITE message according to the service level specified by the user. It is based on the simple priority-scheduling algorithm proposed by this paper.

\section{Performance Analysis}

In order to analyze the performance of differentiated call processing in the SIP6 Server, we implemented a thread test program. We used a Linux system for the SIP6 Server and a windows system for the test program on the IPv6 Testbed in Konkuk University.

We analyzed and compared the performance for call processing time in SIP6 and SIP from Columbia University. Test scenario is as following.

- The test program concurrently sends the INVITE and REGISTER message increasingly of the same number step by step according to the service level to SIP6 Server. In the case of the SIP Server of Columbia University, differentiated service scheme is not applied.

- The test program measures the average of message processing time in establishing a session in the SIP6 and SIP Server.

Fig. 4 presents the test results of average time to process REGISTER message in the SIP6 Registrar Server and SIP Registrar Server. Fig. 5 and Fig. 6 present the test results of average time for processing INVITE messages in the SIP6 and SIP Proxy Server and Redirect Server.

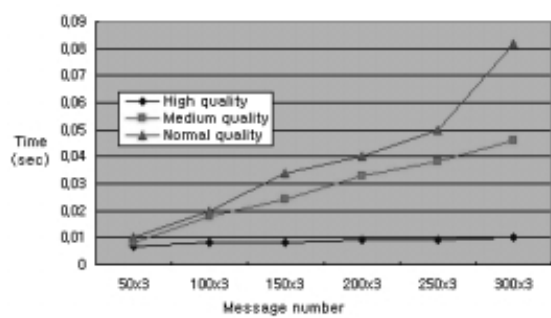

(a) Test result in the SIP6

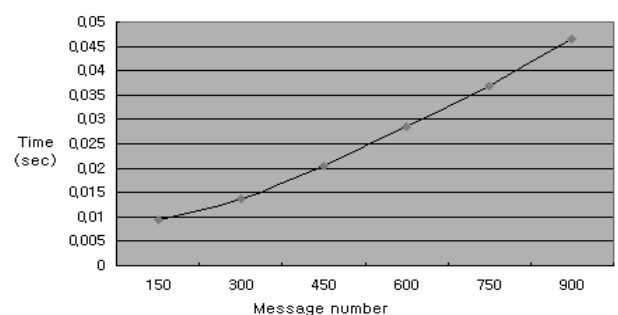

(b) Test result in the SIP

Fig. 4. The average time for processing REGISTER messages in Registrar Server 


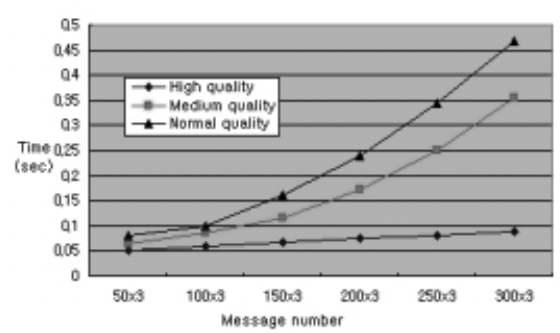

(a) Test result in the SIP6

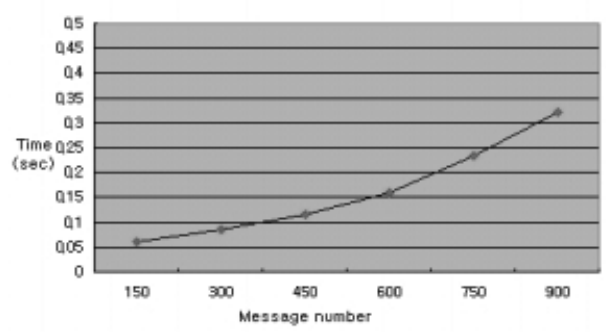

(b) Test result in the SIP

Fig. 5. The average time for processing INVITE messages in Redirect Server

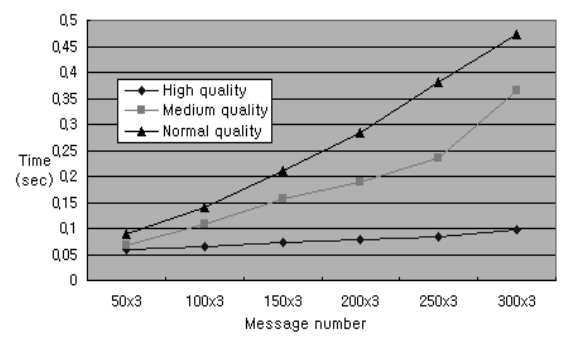

(a) Test result in the SIP6

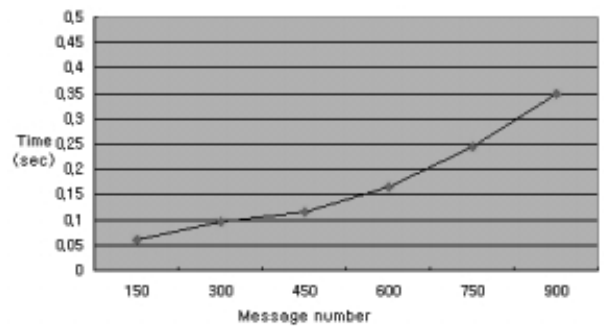

(b) Test result in the SIP

Fig. 6. The average time for processing INVITE messages in Proxy Server

As shown in these results, SIP6 proposed by this paper supports a differentiated call processing service to satisfy the user's required QoS. As INVITE and REGISTER messages increases, the average time in processing messages in the SIP6 Server displays a remarkable difference according to the service levels. Particularly, INVITE and REGISTER message specifying the High Quality always gets the fast service in the call processing.

The results of performance analysis demonstrates that the differentiated call processing service gives better performance for the service requiring a fast session establishment when the network traffic and the requests for session establishment are heavy.

\section{Conclusions and Future Works}

In this paper, we proposed a differentiated call processing mechanism in the SIP6 based on IPv6. Because we implemented an IPv6 in this system, it can use the QoS and the security features of the IPv6. We applied the QoS based on the differentiated service using the simple priority-scheduling algorithm for call processing. We presented that the performance improved. 
In the future, we are going to add an authentication service by using $\mathrm{AH}$ (Authentication Header), ESP (Encapsulation Security Payload) header of IPv6.

\section{References}

1. Handley, H.Schulzrine, E.Schooler, and J.Rosenberg. : SIP: session initiation protocol, RFC 2543 (1999)

2. A.Johnston, S.Donovan, et al. : SIP Telephony Call Flow Examples, Internet Draft (2000)

3. R.Gilligan, S.Thomson, J.Bound, W.Stevens. : Basic Socket Interface Extensions for IPv6, RFC 2553 (1999)

4. R. Hinden, S. Deering. : Internet Protocol version 6(IPv6) specification, RFC 2460 (1998)

5. K. Nichols, S. Blake, F. Baker, D. Black. : Definition of the Differentiated Services Field (DS Field) in the IPv4 and IPv6 Headers, RFC 2474 (1998)

6. P. Ferquson and G. Huston. : Quality of Service, John Wiley \& Sons (1998)

7. S. Donovan, J. Rosenberg. : The SIP Session Timer, Internet Draft (2001)

8. ITU-T Recommendation H.323 (1996)

9. I. Dalgic and H. Fang. : Comparison of H.323 and SIP for IP Telephony Signaling, Photonics East, Proceeding of SPIE'99, Boston, Massachusetts (1999)

10. Henning Schulzrinne and Jonathan Rosenberg. : Signaling for Internet Telephony (1998)

11. S.Blake, M.Carlson, E.Davies, Z.wang, and W.Weiss. : An architecture for differentiated service, Request for Comments (Proposed Standard) 2475, Internet Engineering Task Force (1998)

12. W.Almesberger, T.Ferrari. and J.-Y.Le Boudec. : SRP: a scalable resource reservation protocol for the internet, Tech. Rep. SSC/1998/009, EPFL, Lausanne, Switzerland (1998)

13. P.P.Pan and H.Schulzrinne. : YESSIR: A simple reservation mechanism for the Internet, in Proc. International Workshop on Network and Operating System Support for Digital Audio and Video (NOSSDAV), (Cambridge, England) (1998)

14. Y. Bernet, R. Yavatkar, P.Ford, F.Baker, L. Zhang, M. Speer, and R. Braden. : Interoperation of RSVP/Intserv and diffserv networks, Internet Draft (1999) 